\title{
Deficiencia de vitamina D: ¿Factor de riesgo de síndrome metabólico?
}

\author{
MARVIN ISAAC QUERALES ${ }^{\mathrm{a}}$, MARÍA ELENA CRUCES ${ }^{\mathrm{b}}$, \\ SUSAN ROJAS ${ }^{\text {a }}$, LISSETTE SÁNCHEZ ${ }^{a}$
}

Escuela de Bioanálisis. Facultad de Ciencias de la Salud

Universidad de Carabobo (Valencia, Venezuela).

a Licenciado en Bioanálisis.

${ }^{\mathrm{b}}$ Magíster en Nutrición.

Recibido el 14 de diciembre de 2009, aceptado el 15 de septiembre de 2010

Correspondencia a: Lcdo. Marvin Isaac Querales.

Av. Bolívar Norte, Sector La Ceiba, Callejón Peña-Pérez, Edif. Somos, Apto. 6-1 Valencia, Venezuela Fono. +58 [241] 8380810 Fax: $+58[241] 8561200$ E-mail: marvinquerales@hotmail.com

\section{Association between vitamin D deficiency and metabolic syndrome}

Vitamin D has an essential role in calcium metabolism and bone health. Vitamin D3 or cholecalciferol is synthesized from 7-dehydrocholesterol or provitamin D3, by sunlight ultraviolet radiation to the skin. 7-dehydrocholesterol is subsequently hydroxylated in the liver and then in the kidney to produce 1,25- $(\mathrm{OH}) 2 \mathrm{D} 3$, the active metabolite that binds to specific receptors (VDR) in target tissues, mainly bone and intestine. Other tissues, such as the immune and cardiovascular system, have also VDR. Vitamin D deficiency can induce rickets in children and osteomalacia and osteoporosis in adults. A possible inverse association between vitamin D levels and the prevalence of metabolic syndrome has been proposed. Vitamin D deficiency increases the risk of type 1 diabetes, insulin resistance, and hypertension, key components of this syndrome. However, other studies have not confirmed this association. Further clinical and experimental studies are needed to ascertain the role of vitamin D in metabolic syndrome.

(Rev Med Chile 2010; 138: 1312-1318).

Key words: Insulin resistance; Vitamin D; Metabolic syndrome X.
S e ha reportado un notable incremento en investigaciones que sugieren el papel transcendental de la vitamina $\mathrm{D}$ en las funciones metabólicas del cuerpo humano. Está muy bien estudiado su rol primario en el mantenimiento de la homeostasia del calcio y del fósforo, bien sea por incremento en la absorción de calcio intestinal o por su actividad en el metabolismo óseo ${ }^{1}$.

Sin embargo, la vitamina D también ejerce efectos sobre el sistema inmunológico, microendocrino de la vasculatura y en la prevención de distintos tipos de cáncer ${ }^{2}$. Sus acciones a nivel del sistema cardiovascular están fundamentadas en estudios que sugieren la ingesta de vitamina D para prevenir patologías, tales como la aterosclerosis $^{3}$, hipertensión ${ }^{4}$, resistencia a la insulina ${ }^{5}$ e hiperglicemia ${ }^{6}$; factores de riesgos esenciales en la aparición del síndrome metabólico. El presente es un artículo de revisión que tiene como objetivo valorar las acciones no clásicas de la vitamina $\mathrm{D}$ que están involucradas en la génesis de esta condición clínica que eleva el riesgo de sufrir un evento cardiovascular.

Vitamina D: Síntesis, metabolismo y funciones biológicas clásicas

La vitamina $\mathrm{D}_{3}$ o colecalciferol proviene del 7-dehidrocolesterol o provitamina $\mathrm{D}_{3}$, compuesto producido en grandes cantidades en la piel de muchos animales vertebrados, incluyendo los humanos. Durante la exposición a los rayos solares, el 7-dehidrocolesterol absorbe la radiación ultravioleta B (UVB), con una longitud de onda entre 290-315 nm. La misma conlleva a un rearreglo del 5,7-dieno de la provitamina $\mathrm{D}_{3}$, rompiendo así el anillo B entre los carbonos 9 y 10 
y dando lugar a un trieno 6,7-cis que se conjuga para formar un 9,10-secolesterol conocido como previtamina $\mathrm{D}_{3}$. La previtamina $\mathrm{D}_{3}$ termodinámicamente inestable, se isomeriza inmediatamente a vitamina $\mathrm{D}_{3}$ mediante un proceso dependiente de temperatura ${ }^{7}$.

Una vez formada, la vitamina $\mathrm{D}_{3}$, estructuralmente incompatible con las cadenas hidrofóbicas de los ácidos grasos de la membrana plasmática, es expulsada hacia el espacio extracelular y se une a una proteína $\alpha-2$ globulina específica de grupo (Gc) sintetizada en el hígado, conocida como proteína de unión a vitamina $\mathrm{D}(\mathrm{DBP})$ o transcalciferrina. En caso de existir una persistencia de la radiación $\mathrm{UV}$, la previtamina $\mathrm{D}_{3}$ no se isomeriza a vitamina $\mathrm{D}_{3}$ sino que lo hace a taquisterol o luministerol, en un mecanismo protector del exceso de formación de vitamina $\mathrm{D}_{3}$.

Enlazada a la DPB, la vitamina $\mathrm{D}_{3}$ es transportada al hígado (y también a otros tejidos), donde sufre su primera hidroxilación en el carbono 25 catalizada por la enzima 25-hidroxilasa hepática dando como resultado la principal forma circulante de la vitamina $\mathrm{D}_{3}$, la 25-hidroxivitamina $\mathrm{D}_{3}$ (25- $\mathrm{OHD}_{3}$ ) o calcidiol. Este metabolito tiene una prolongada vida media y es considerado como el índice de estado nutricional en vitamina $\mathrm{D}$.

Aunque $25-\mathrm{OHD}_{3}$ sea la principal forma circulante de vitamina $\mathrm{D}$, biológicamente es inerte y para activarse requiere de otra hidroxilación, esta vez en el carbono 1 para formar $1,25-(\mathrm{OH})_{2} \mathrm{D}_{3}$ o calcitriol por acción de la enzima $25-\mathrm{OHD}_{3}{ }^{3}$ $1 \alpha$-hidroxilasa $(\mathrm{CYP} 1 \alpha)$, enzima oxidasa mitocondrial que se encuentra presente en las células tubulares renales y en otros tipos de células. La actividad de la CYP1 $\alpha$ renal es estimulada por la hormona paratiroidea (PTH) e inhibida por niveles elevados de calcio. La producción extrarrenal se estimula por citoquinas (interferón $\gamma$ y TNF, preferentemente) más que por la $\mathrm{PTH}$ y resulta poco inhibida por el calcio.

La enzima 24-hidroxilasa (CYP24) es responsable de la hidroxilación en posición 24 de la 25$\mathrm{OHD}_{3}$ y $1,25-(\mathrm{OH})_{2} \mathrm{D}_{3}$ para formar $24,25-(\mathrm{OH})_{2} \mathrm{D}_{3}$ y $1,24,25-(\mathrm{OH})_{3} \mathrm{D}_{3}$, respectivamente. Se expresa fundamentalmente en el túbulo renal pero su distribución tisular es mucho más amplia (en general donde existen receptores para calcitriol). $\mathrm{La}$ afinidad por la $1,25-(\mathrm{OH})_{2} \mathrm{D}_{3}$ es más alta que por la $25-\mathrm{OHD}_{3}$, por lo que se ha considerado que esta enzima constituye un eficiente mecanismo para eliminar el exceso y toxicidad de la $1,25-(\mathrm{OH})_{2} \mathrm{D}_{3}$.

La principal función biológica de la vitamina $\mathrm{D}$ es mantener las concentraciones intra y extracelulares de calcio en un margen fisiológicamente aceptable. Esto se logra mediante translocación de 1,25- $(\mathrm{OH})_{2} \mathrm{D}_{3}$ dentro de la célula, en donde se une a un receptor de vitamina $\mathrm{D}$ de alta afinidad (VDR), miembro de la superfamilia de receptores nucleares. Los VDR se distribuyen ampliamente y no se encuentran restringidos en tejidos dianas clásicos de la vitamina $\mathrm{D}$, lo que justifica la variedad de acciones del calcitriol en el organismo ${ }^{7}$. El VDR, luego de unirse al $1,25-(\mathrm{OH})_{2} \mathrm{D}_{3}$, se heterodimeriza con otros receptores hormonales, particularmente con la familia de los receptores retinoides X. Este complejo se liga a secuencias de ADN, llamados elementos de respuesta a la vitamina D (VDRE) ${ }^{8}$, lo que da como resultado la transcripción de ARNm específico de hormona que gobierna la traducción de varias proteínas, entre ellas la de unión a calcio. Se cree que esta proteína es importante en el transporte transcelular de calcio en el intestino. El resultado neto consiste en aumentar la absorción de calcio y fósforo desde el contenido intestinal hasta la circulación. Por otro lado, el $1,25-(\mathrm{OH})_{2} \mathrm{D}_{3}$ tiene varios efectos en las células ósea, principalmente incrementar la movilización de calcio y las reservas de fósforo en el hueso cuando hay hipocalcemia (Figura 1).

\section{Deficiencia de vitamina $\mathrm{D}$ en relación con algunos factores de riesgo para síndrome metabólico}

Aunque están muy bien caracterizadas las consecuencias que tiene la deficiencia de vitamina D sobre el sistema óseo, investigaciones recientes sugieren que niveles disminuidos de esta vitamina producen efectos adversos en el sistema cardiovascular; siendo más evidente en individuos con hipertensión y en aquellos con niveles de vitamina $\mathrm{D}<15 \mathrm{ng} / \mathrm{mL}^{9}$. De hecho, estudios prospectivos han encontrado que la hipovitaminosis D aumenta notablemente la disfunción miocárdica y el consecuente fallo cardíaco ${ }^{10} \mathrm{y}$ mortalidad cardiovascular en paciente con hemodiálisis ${ }^{11}$.

El síndrome metabólico combina una serie de factores genéticos y asociados al estilo de vida que aumentan la predisposición a eventos cardiovasculares. Entre estos se encuentran la hiperglicemia, la 


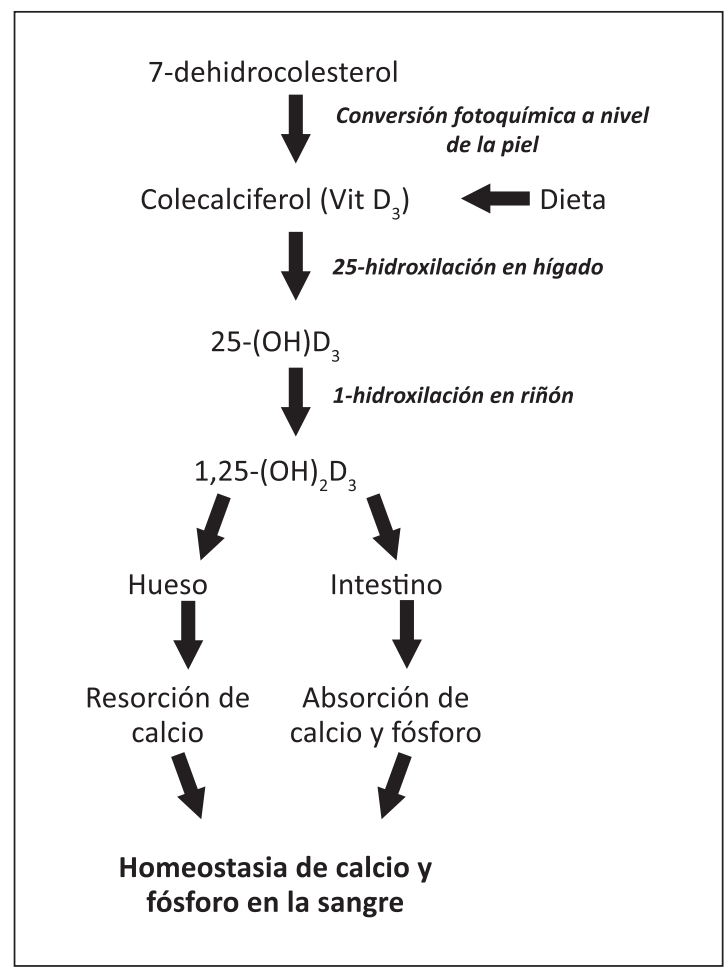

Figura 1. Metabolismo de la vitamina D y sus funciones biológicas clásicas.

hipertensión arterial, la dislipidemia y la obesidad abdominal, los cuales pudieran estar relacionados (directa o indirectamente) con la deficiencia de vitamina $\mathrm{D}$.

\section{Diabetes mellitus:}

Existe evidencia que destaca el rol fundamental que desempeña la vitamina $\mathrm{D}$ en la secreción normal de insulina, incluyendo un efecto directo sobre los VDR en las células $\beta$ y uno indirecto mediado por la existencia de proteínas fijadoras de calcio dependientes de vitamina $\mathrm{D}$ en los tejidos pancreáticos ${ }^{12}$. De hecho, se ha reportado que pacientes deficientes de vitamina $D$ y con limitada secreción de insulina, muestran una mejora en la síntesis de ésta última una vez que la vitamina es suplementada en la dieta ${ }^{5}$. El mismo es consistente con otras investigaciones en donde aseguran el efecto benéfico que tiene la $1,25-(\mathrm{OH})_{2} \mathrm{D}_{3}$ sobre la función celular beta en animales en experimentación ${ }^{13}$.

La influencia ejercida por la vitamina D sobre la secreción de insulina puede seguir varias vías.
Esta vitamina estimula las células $\beta$ mediante un incremento en las concentraciones intracelulares de calcio a través de unos canales de voltaje no selectivos ${ }^{14}$, produciendo una activación de las endopeptidasas dependientes de calcio dando lugar a un clivaje que facilita la conversión de proinsulina a insulina ${ }^{5,13}$. El calcio no sólo es necesario para la biosíntesis de insulina, sino también para la glicólisis de las células $\beta$, siendo esencial en la señalización de la concentración de glucosa circulante ${ }^{15}$. La vitamina D también ejerce efectos en la secreción de insulina por estimulación de su síntesis mediante la activación de proteínas a nivel de los islotes pancreáticos ${ }^{16}$.

En comparación con los estudios sobre disfunción de las células $\beta$, la relación entre los niveles disminuidos de vitamina $\mathrm{D}$ y la sensibilidad a la insulina es bastante limitada. Una asociación positiva fue reportada en un grupo de 34 hombres, incluyendo 7 sujetos con diabetes ${ }^{17}$; además la suplementación con vitamina $\mathrm{D}$ reduce las concentraciones de ácidos grasos libres en suero, lo cual sugiere un incremento en la sensibilidad a la insulina ${ }^{18}$. En base a esto se ha postulado que la deficiencia de vitamina $\mathrm{D}$ puede conllevar al desarrollo de diabetes tipo 2.

La diabetes tipo 2 se caracteriza por la presencia de alteraciones en la secreción de insulina y defectos en su reconocimiento (resistencia), trayendo consigo intolerancia a la glucosa y un aumento de su síntesis endógena. Recientemente la hipovitaminosis $\mathrm{D}$ se ha señalado como factor de riesgo para la intolerancia a la glucosa, encontrándose un aumento en la secreción de insulina y una mejora considerable en la tolerancia a la glucosa en pacientes que reciben tratamiento suplementado con vitamina $\mathrm{D}^{19-20}$ y estableciéndose, por tanto, una asociación inversa entre los niveles de vitamina $\mathrm{D}$ y el riesgo a desarrollar diabetes tipo $2^{21}$. La inflamación sistémica asociada con la diabetes tipo 2, mediada por citoquinas que inducen la apoptosis de las células $\beta$, también puede ser modulada por los efectos de esta vitamina.

Cabe destacar que la prevalencia de este tipo de diabetes es elevada en pacientes con obesidad, la cual se relaciona con la hipovitaminosis $\mathrm{D}$, debido que la vitamina es eficientemente depositada en las fuentes de almacenamiento de grasa corporal donde no es completamente biodisponible ${ }^{7,19}$. Esto resulta curioso, ya que al ser la obesidad abdominal un factor de riesgo para síndrome metabólico, la 
misma pudiera coadyuvar a la disminución de los niveles séricos de $25(\mathrm{OH}) \mathrm{D}$ y traería consigo resistencia a la insulina. Aunque esta hipótesis sería una explicación del por qué los pacientes a la larga pudieran desarrollar diabetes tipo 2, está lejos de ser comprendida en su totalidad.

La deficiencia de vitamina $\mathrm{D}$ es más común en pacientes con diabetes tipo 2 que con diabetes tipo $1^{22}$. No obstante, su posible rol en la génesis de esta última se ha dilucidado con mayor profundidad. En la diabetes mellitus tipo 1, la $1,25-(\mathrm{OH})_{2} \mathrm{D}_{3}$, tiene notables efectos antiinflamatorios e inmunomoduladores que podrían ser de utilidad en su tratamiento.

En condiciones experimentales, la vitamina D inhibe la proliferación y la función citotóxica de los linfocitos T. Cuando se añade a cultivos de células mononucleares periféricas (PMBCS), el 1,25- $(\mathrm{OH})_{2} \mathrm{D}_{3}$ disminuye la proliferación y la síntesis de inmunoglobulinas y de citoquinas, que incluyen a la interleukina-1 (IL-1), IL-2, IL-6, IL12 , factor de necrosis tumoral alfa (TNF- $\alpha$ ) e interferón- $\gamma($ IFN- $\gamma)$, lo que implica una regulación de la respuesta Th1 $1^{23-24}$. Sin embargo, la 1,25- $(\mathrm{OH})_{2} \mathrm{D}_{3}$ también promueve la producción de IL-4, IL-5 e IL-10, con lo cual promueve la activación de la respuesta $\mathrm{Th} 2^{25}$. Gracias a este cambio de respuesta de las células $T$, se protege a las células beta de la lesión pancreática orquestada por las citoquinas de la respuesta Th1 ${ }^{26}$. El 1,25- $(\mathrm{OH})_{2} \mathrm{D}_{3}$ tiene la capacidad de disminuir la actividad presentadora de antígenos de los macrófagos hacia los linfocitos mediante la reducción de la expresión de moléculas del complejo mayor de histocompatibilidad tipo II (MCH-II) en la superficie celular ${ }^{27}$. Además, también podría proteger contra el desencadenamiento de la diabetes tipo 1, a través del estímulo y reclutamiento de las células T reguladoras CD4+ y CD8+ en el sitio de la lesión pancreática ${ }^{28}$. Todas estas acciones en conjunto, tiene como objetivo disminuir la respuesta inflamatoria y la inmunidad mediada por células propias de esta enfermedad.

\section{Hipertensión arterial}

Existe evidencia, a partir de varios modelos animales, que la vitamina $\mathrm{D}$ es importante en el control de la presión sanguínea y la hipertensión arterial $^{29}$. Krause et $\mathrm{al}^{30}$ reportaron que pacientes hipertensos expuestos a radiación ultravioleta B (UVB) por más de 3 meses tuvieron más de $180 \%$ de incremento en las concentraciones de
$25-\mathrm{OHD}_{3}$ circulante y un descenso de $6 \mathrm{mmHg}$ en sus presiones sanguíneas diastólicas y sistólicas, resultados similares a los esperados si los pacientes hubiesen recibido tratamiento farmacológico para la hipertensión. El mecanismo exacto por el cual la radiación UVB mejora la presión sanguínea en estos pacientes adultos aún no está muy bien dilucidada. Una hipótesis interesante es la propuesta por Li et $\mathrm{al}^{31} \mathrm{y}$ por Xiang et $\mathrm{al}^{32}$, en donde, basados en estudios experimentales, indican que $1,25(\mathrm{OH})_{2} \mathrm{D}_{3}$ participa en la regulación del sistema renina-angiotensina por supresión directa de la expresión del gen. De hecho, la sobreexpresión de renina puede ser producida en ratones salvajes mediante inhibición farmacológica de la síntesis de vitamina $\mathrm{D}^{31}$.

Otra propuesta que se maneja, es aquella que involucra la posible influencia de la vitamina D sobre el tono vascular y la hemodinámica cardiovascular, observándose que la vitamina $\mathrm{D}$ causa cambios rápidos en los pacientes con hipertensión esencial, pero no en los controles, lo que incrementa la resistencia vascular ${ }^{33}$. Estos resultados están soportados en ensayos experimentales, en donde el 1,25- $(\mathrm{OH})_{2} \mathrm{D}_{3}$ aumenta la sensibilidad de la resistencia arterial a la norepinefrina en ratas hipertensas, pero no en las normotensas, $y$ rápidamente mejora la fuerza arterial generada por modulación de la concentración intracelular de calcio ${ }^{29,34}$.

\section{Dislipidemia}

Son pocos los estudios que relacionan niveles deficientes de vitamina $\mathrm{D}$ con hipercolesterolemia, ya que al parecer la ingesta de calcio tiene una implicación más directa. En este sentido, fue reportado que personas con sobrepeso y con un régimen alimenticio bajo en calcio presentan un mayor riesgo de padecer síndrome metabólico que aquellas personas, igualmente con sobrepeso, que tengan una dieta rica en este mineral ${ }^{35}$. Fujita y Palmieri ${ }^{36}$ propusieron una relación paradójica en donde niveles bajos de calcio, en concordancia con un contenido subóptimo de calcio a nivel óseo, pudieran estar asociados con su incremento en los tejidos blandos. En base a esto, Zemel et $\mathrm{al}^{37}$ demostraron que una dieta hipocalcémica conllevan a un alto contenido del calcio en los adipocitos, trayendo consigo un incremento de la lipogénesis y una reducción de la movilización de los lípidos, lo cual pudiera explicar la asociación reportada por 
Melanson et $\mathrm{al}^{38}$ entre una poca ingesta de calcio y una baja oxidación de lípidos en humanos.

Recientemente, se ha estado evaluando el posible efecto de la vitamina D sobre la expresión de genes de apoliproteínas ${ }^{39}$, pero los resultados siguen siendo controversiales.

\section{Relación deficiencia de vitamina D - síndrome metabólico}

La vitamina $\mathrm{D}$ se ha asociado fuertemente con factores de riesgo individuales que condicionan el síndrome metabólico (SM) (Figura 2). Sin embargo, son muchas las dudas en cuanto a la posible relación entre este síndrome y la hipovitaminosis D. Investigaciones sugieren una asociación inversa entre los niveles de vitamina $\mathrm{D}$ y la presencia de $\mathrm{SM}^{5,40}$, siendo consistente con los numerosos efectos que ejerce esta vitamina sobre una gran cantidad de tejidos y que fueron explicados con anterioridad en este artículo. Estos mismos estudios reportan una probabilidad 54\% más baja de padecer SM en individuos con niveles de vitamina $D$ adecuados ${ }^{5} y$ un aumento de aproximadamente tres veces la prevalencia de SM en individuos vitamina D- deficientes ${ }^{40}$. No obstante, estos estudios difieren de los encontrados por Reis et $\mathrm{a}^{41}$ quienes no evidenciaron asociación inversa entre los niveles de $25-\mathrm{OHD}_{3}$ y la presencia de $\mathrm{SM}$, pero sí observaron un incremento de la probabilidad de esta entidad clínica con un aumento de los niveles de la hormona paratiroidea (PTH) en hombre. Resultados similares fueron obtenidos por Hjelmesaeth et $\mathrm{al}^{42}$, quienes afirman igualmente que los niveles de PTH, más no de Vitamina D, son un factor de morbilidad para SM en hombres y mujeres obesas. De la misma manera, Liu et $\mathrm{al}^{43}$ tampoco muestran una asociación inversa entre la ingesta de vitamina D y el SM, ratificando el papel esencial del calcio en la prevención del mencionado síndrome. Por tanto, la relación SM-deficiencia de vitamina $\mathrm{D}$ sigue siendo incierta.

\section{Conclusión}

La deficiencia de vitamina $\mathrm{D}$ y sus consecuencias constituye un problema de salud pública a nivel mundial, que afecta principalmente a aquellos países ubicados a elevadas latitudes como Argentina y Chile, siendo la principal causa la falta de exposición solar. En Argentina, específicamente en regiones australes, los niveles séricos de vitamina $\mathrm{D}$ disminuyen significativamente en período invernal ${ }^{44}$. Por su parte, en Chile no existen mediciones de grandes cohortes de chilenos para conocer su estado nutricional de vitamina $\mathrm{D}$ debido a que la incidencia detectada depende del punto de corte en los niveles séricos de 25-OHD

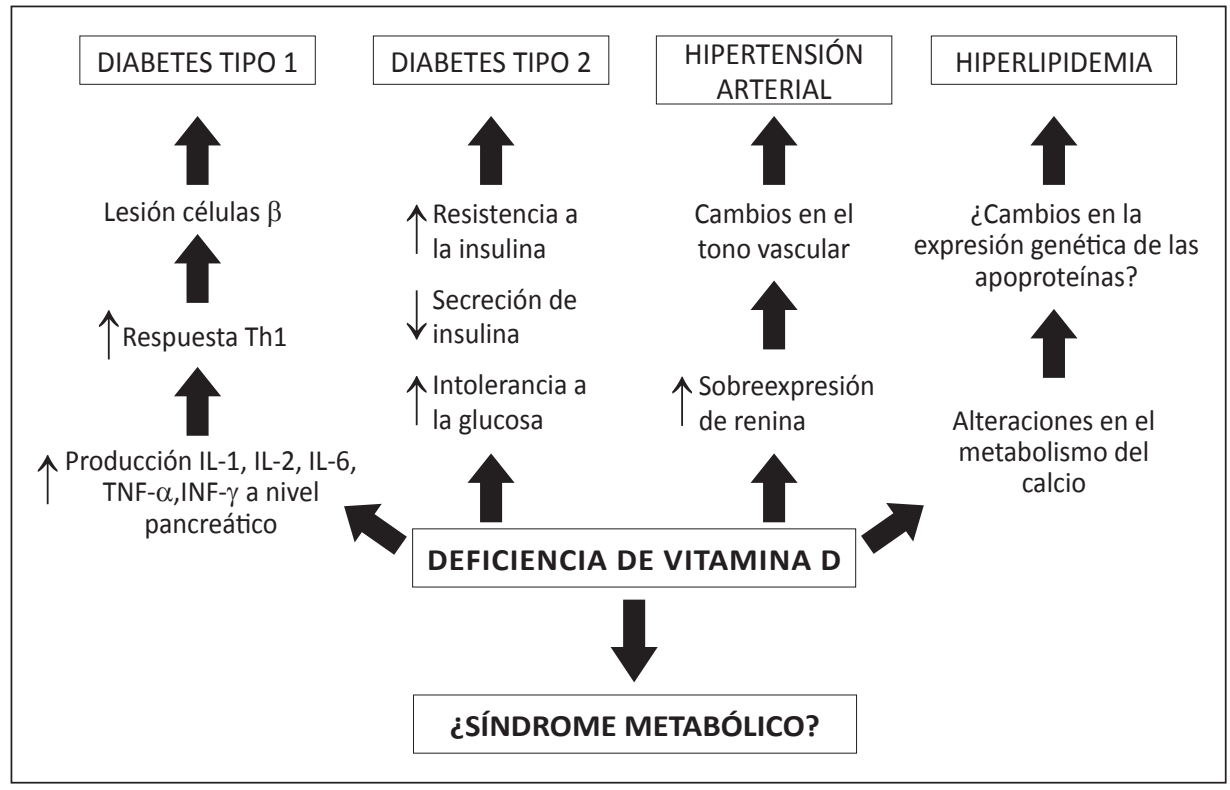

Figura 2. Papel de la vitamina $D$ en el desarrollo de los factores de riesgo que condicionan el síndrome metabólico. 
para que sea considerada como deficiencia. Sin embargo, en distintos grupos de estudio, tales como adultos mayores ${ }^{45}$ y mujeres de edad media (obesas operadas de bypass gástrico y controles) ${ }^{46}$ se han comprobado que, la frecuencia de déficit alcanza prácticamente $85 \%$ de la muestra, especialmente en los meses de invierno.

Estas cifras alarmantes, conllevan a expandir el espectro de abordaje de las consecuencias de la hipovitaminosis $\mathrm{D}$, no sólo las óseas sino también las relacionadas con sus acciones no clásicas. De ahí que es preciso que se lleven a cabo un mayor número de investigaciones que determinen si existe una asociación directa entre los niveles séricos de vitamina $\mathrm{D}$ y el riesgo a padecer SM; sobre todo en países latinoamericanos, donde la prevalencia de SM está alcanzando cifras alarmantes como las de Estados Unidos de Norteamérica y Europa. Son pocos los trabajos que se enfocan en estudiar la etiología del SM relacionada con la deficiencia de vitamina $\mathrm{D}$, aun cuando existen evidencias de que su suplementación puede ser beneficiosa para la optimización de la respuesta en todos los procesos asociados al SM. Se hace necesario la comprensión y explicación de los mecanismos exactos que conllevan a la aparición de este síndrome y analizar la complementariedad de cada uno de los factores de riesgo.

\section{Referencias}

1. Rajakumar K. Vitamin D, cod-liver oil, sunlight, and rickets: a historical perspective. Pediatrics 2003; 112: 132-5.

2. Pozzo, M. Vitamina D: Acciones no clásicas. Actualizaciones en osteología 2005; 1: 28-33.

3. Moon J, Bandy B, Davison A. Hypothesis: etiology of atherosclerosis and osteoporosis: are imbalances in the calciferol endocrine system implicated? J Am Coll Nutr 1992; 11: 567-83.

4. Pfeifer M, Begerow B, Minne H, Nachtigall D, Hansen C. Effects of a short-term Vitamin $\mathrm{D}_{3}$ and calcium supplementation on blood pressure and parathyroid hormone levels in elderly women. J Clin Endocrinol Metab 2001; 86: 1633-7.

5. Chiu K, Chu A, Go V, Saad M. Hypovitaminosis D is associated with insulin resistance and beta cell dysfunction. Am J Clin Nutr 2004; 79: 820-5.

6. Scragg R, Sowers M, Bell C. Serum 25-hydroxyvitamin $\mathrm{D}$, diabetes, and ethnicity in the third national health and nutrition examination survey. Diabetes care 2004; 27: 2813-8.

7. Holick M. Vitamin D: importance in the prevention of cancers, type 1 diabetes, heart disease and osteoporosis. Am J Clin Nutr 2004; 79: 362-71.

8. Barsony J, Prufer K. Vitamin D receptor and retinoid X receptor interactions in motion. Vitamin Horm 2002; 65: 345-76.

9. Wang TJ, Peencina MJ, Booth SL, Jacquees PF, Ingelson E, Lanier K, et al. Vitamin D deficiency and risk cardiovascular Disease. Circulation 2008; 117: 503-11.

10. Pilz S, Marz W, Wellnitz B, Seelhorst U, FahrleitnerPammer A, Dimai HP, et al. Association of Vitamin D Deficiency with Heart Failure and Sudden Cardiac Death in a Large Cross-Sectional Study of Patients Referred for Coronary Angiography. J Clin Endocrinol Metab 2008; 93: 3927-35.

11. Wolf M, Shah A, Gutiérrez O, Ankers E, Monroy M, Tamez H, et al. Vitamin D levels and early mortality among incidnt hemodialysis patients. Kidney Int 2007; 72: 1004-13.

12. Johnson JA, Grande JP, Roche PC, Kumar R. Immunohistochemical localization of the $1,25(\mathrm{OH})_{2} \mathrm{D}_{3}$ receptor and calbindin D28K in human and rat pancreas. Am J Physiol 1994; 267: E356-60.

13. Ayesha I, Bala T, Reddy C, Raghuramulu N. Vitamin D deficiency reduces insulin secretion and turnover in rats. Diabetes Nutr Metab 2001; 14: 78-84.

14. Sergeev IN, Rhoten WB. 1,25-Dihydroxyvitamin D3 evokes oscillations of intracellular calcium in a pancreatic b-cell line. Endocrinology 1995; 136: 2852-61.

15. Boucher BJ. Inadequate vitamin D status: does it contribute to the disorders comprising syndrome " $\mathrm{X}$ ". Br J Nutr 1998; 79: 315-27.

16. Borissova AM, Tankova T, Kirilov G, Dakovska L, Kovacheva $\mathrm{R}$. The effect of vitamin D3 on insulin secretion and peripheral insulin sensitivity in type 2 diabetic patients. Int J Clin Pract 2003; 57: 258-61.

17. Lind L, Heanni A, Litthell H, Hvarfner A, Seorensen OH, Ljunghall S. Vitamin D is related to blood pressure and other cardiovascular risk factors in middle-aged men. Am J Hypertens 1995; 8: 894-901.

18. Inomata S, Kadowaki S, Yamatani T, Fukase M, Fujita T. Effect of 1 alfa (OH)-vitamnin D3 on insulin secretion in diabetes mellitus. Bone Miner 1986; 1: 187-92.

19. Zittermann A. Vitamin D in preventive medicine: are we ignoring the evidence? Br J Nutr 2003; 89: 552-72.

20. Pittas AG, Harris SS, Stark PC, Dawson-Hughes B. The effects of calcium and vitamin D supplementation on blood glucose and markers of inflammation in nondiabetic adults. Diabetes Care 2007; 30: 980-6. 
Vitamina D y síndrome metabólico - M. I. Querales et al

21. Mattila C, Knekt P, Männistö S, Rissanen H, Laaksonen MA, Montonen J, et al. Serum 25-Hydroxyvitamin D concentration subsequent risk of Type 2 diabetes. Diabetes Care 2007; 30: 2569-70.

22. Di Cesar, DJ, Ploutz-Snyder R, Weinstock RS, Moses AM. Vitamin D deficiency is more common in type 2 than in type 1 diabetes. Diabetes Care 2006; 29: 174.

23. Becker B, Hullet D, O'Herrin J, Malin G, Sollinger H, DeLuca H. Vitamin D as inmunomodulatory therapy for kidney transplantation. Transplantation 2002; 74: 1204-6.

24. Lemire J, Archer D, Beck L, Spiegelber H. Immunosuppressive actions of 1,25-dihydroxyvitamin D3: preferential inhibition of Th1 functions. J Nutr 1995; 125: 1704S-08S.

25. Long K, Santos J. Vitamins and the regulation of the immune response. Pediatr Infect Dis J 1999; 18: 283-90.

26. Adorini L. Tolerogenic dendritic cells induced by vitamin $\mathrm{D}$ receptor ligands enhance regulatory $\mathrm{T}$ cells inhibiting autoimmune diabetes. Ann NY Acad Sci 2003; 987: 258-61.

27. Rigby W, Waugh M, Graziano R. Regulation of human monocyte HLA-DR and CD4 antigen expression, and antigen presentation by 1,25-dihidroxyvitamin D3. Blood 1990; 76: 189-97.

28. Zella J, DeLuca H. Vitamin D autoimmune diabetes. J Cell Biochem 2003; 88: 216-22.

29. Bian K, Ishibashi $\mathrm{K}$, Bukoski R. $1,25(\mathrm{OH})_{2} \mathrm{D}_{3}$ modulates intracellular $\mathrm{Ca}^{+2}$ and force generation in resistance arteries. Am J Physiol 1996; 270: H230-7.

30. Krause R, Buhring M, Hopfenmuller W, Holick M, Sharma A. Ultraviolet B and blood pressure. Lancert 1998; 352: 709-10.

31. Li Y, Kong J, Wei M, Chen Z, Liu S, Cao L. 1,25-dihydroxyvitamin $\mathrm{D}_{3}$ is a negative endocrine regulator of rennin-angiotensin system. J Clin Invest 2002; 110: 229-38.

32. Xiang W, Kong J, Chen S, Cao L, Qiao G, Zheng W, et al. Cardiac hypertrophy in vitamin D receptor Knockout mice: role of the systemic and cardiac rennin-angiotensin systems. Am J Physiol Endocrinol Metab 2005; 228 : E125-32.

33. Jespersen B, Randlow A, Abrahamsen J, Fogh-Andersen N, Olsen N, Kanstrup I. Acute cardiovascular effect of 1,25-dihydroxycholecalciferol in essential hypertension. Am J Hypertrns 1998; 11: 659-66.

34. Hatton D, Xue H, DeMerritt H, McCarron D. 1,25$(\mathrm{OH})_{2}$ Vitamin $\mathrm{D}_{3}$-induced alterations in vascular reactivity in the spontaneously hypertensive rats. Am J Med Sci 1995; 269: C443-50.
35. Pereira M, Jacobs D, Van Horn L, Slattery M, Kartashov A, Ludwig D. Dairy consumption, obesity, and the insulin resistance syndrome in young adults: the CARDIA study. JAMA 2002; 287: 2081-9.

36. Fujita T, Palmieri G. Calcium Paradox disease: calcium deficiency prompting secondary hyperparathyroidism and cellular calcium overload. J Bone Miner Metab 2000; 18: 109-25.

37. Zemel M, Shi H, Greer B, Dirienzo D, Zemel P. Regulation of adiposity by dietary calcium. FASEB J 2000; 14 : 1132-8.

38. Melanson E, Sharp T, Schneider J, Donahoo W, Grunwald G, Hill J. Relation between calcium intake and fat oxidation in adult humans. Int J Obes Relat Metab Disord 2003; 77: 1448-52.

39. Wehmeir $\mathrm{K}$, Beers $\mathrm{A}$, Haas $\mathrm{M}$, et al. Inhibition of AI gene expression by 1,25-dihydroxyvitamin D3. Biochim Biophys Acta 2005; 1737: 16-26.

40. Ford E, Ajani U, McGuire L, Liu S. Concentrations of serum vitamin $\mathrm{D}$ and the metabolic syndrome among U.S. adults. Diabetes Care 2005; 28: 1228-30.

41. Reis J, von Mühlen D, Kritz-Silverstein D, Wingard D, Barrett-Connor E. Vitamin D, Parathyroid hormone levels, and the prevalence of Metabolic Syndrome in community-Dwelling older adults. Diabetes Care 2007; 30: 1549-55.

42. Hjelmesaeth J, Hofs $\varnothing$ D, Aasheim ET, Jenssen T, Moan J, Hager $\mathrm{H}$, et al. Parathyroid hormone, but not vitamin D, is associated with the metabolic syndrome in morbidly obese women and men: a cross-sectional study. Cardiovascular Diabetology 2009; 8: 7

43. Liu S, Song Y, Ford E, Manson J, Buring J, Ridker P. Dietary calcium, vitamin D, and the prevalence of Metabolic Syndrome in Middle-Aged and Older U.S. Women. Diabetes Care 2005; 28: 2926-32.

44. Oliveri MB, Ladizesky M, Mautalen CA, Alonso A, Martínez L. Seasonal variations of hydroxyvitamin D and parathyroid hormone in Ushuaia (Argentina), the southern most city of the world. Bone Miner 1993; 20: 99-108

45. Bunout D, Barrera G, Leiva L, Gattas V, de la Maza MP, Haschke F, et al. Effect of a nutritional supplementation on bone health in Chilean elderly subjects with femoral osteoporosis. J Am Coll Nutr 2006; 25: 170-7.

46. De la Maza MP, Leiva L, Barrera G, Boggiano C, Herrera T, Pérez Y, et al. Evaluación a largo plazo del estado nutricional, composición corporal y densidad mineral ósea en mujeres operadas de bypass gástrico: impacto del nivel socioeconómico. Rev Med Chile 2008; 136: 1415-23. 